


\title{
Isolation, Characterisation and In Silico Toxicity Evaluations of Thiocarbamates, Isothiocyanates, Nitrile, Glucosinolate and Lipids from Moringa oleifera Lam. Seed
}

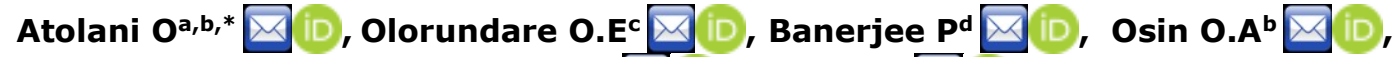 \\ Preissner R $\mathrm{R}^{\mathrm{d}}$ (D), Njan A.A $\mathrm{Q}$ (D) \\ ${ }^{a}$ Department of Chemistry, University of Ilorin, P.M.B. 1515, Ilorin, Nigeria. \\ ${ }^{b}$ Department of Chemical Sciences, Redeemer's University, Ede, Osun State, Nigeria. \\ ${ }^{c}$ Department of Pharmacology and Therapeutics, University of Ilorin, P.M.B. 1515, Ilorin, Nigeria. \\ 'Structural Bioinformatics Group, Institute for Physiology, Charité - University Medicine Berlin, Berlin, \\ Germany.
}

\begin{abstract}
The phytochemical composition of medicinal plants is responsible for the ethnopharmacological applications. These phytochemicals vary in plants of same species planted in various geographical locations. The seed of Moringa oleifera Lam., a widely consumed multi-medicinal plant was examined for the bioactive phytochemicals. With the aid of Nuclear Magnetic Resonance $\left({ }^{1} \mathrm{H}-\right.$ NMR $/{ }^{13} \mathrm{C}-\mathrm{NMR}$ ) spectrometer, Electrospray Ionisation Mass Spectrometry (ESI-MS) and Fourier Transform Infrared (FT-IR) spectroscopy, the phytochemical investigation of the seed of $\mathrm{M}$. oleifera afforded nine compounds which included niazimicin 1, niazidin 2, glucomoringin isothiocyanate 3, niazinin acetate $\mathbf{4}$, niazinin triacetate $\mathbf{5}$, niazirinin $\mathbf{6}$, glucotropaeolin $\mathbf{7}$, triolein $\mathbf{8}$, trivaccenin $\mathbf{9}$. The Gas Chromatography-Mass Spectrometry (GC-MS) analysis of a major oily fraction revealed the presence of additional ten compounds which include oleic acid (major), 13-docosenoic acid, stearic acid, p-hydroxybenzyl cyanide, a -I-rhamnopyranose and other fatty acids/esters. This is the first account of a fully acetylated niazinin (a 4-(2',3',4'-O-triacetyl-a-L-rhamnosyloxy)benzyl isothiocyanate) 5 from Moringa oleifera seed. The in silico toxicity evaluation indicated that most of the isolated compounds are either immunotoxic, carcinogenic or mutagenic. The result thus obtained could serve a basis for the pharmacological and toxicological evaluation and profiling of the seed. The result further implied the need for dosage regulation of the consumption of the seed.
\end{abstract}

Keywords: Moringa oleifera; Niazimicin; Niazidin; Isothiocyanate; Glucosinolate; Nitrile.

Submitted: May 24, 2019. Accepted: December 12, 2019.

Cite this: Atolani $O$, Olorundare $O E$, Banerjee $P$, Osin $O A$, Preissner $R$, Njan $A A$. Isolation, Characterisation and In Silico Toxicity Evaluations of Thiocarbamates, Isothiocyanates, Nitrile, Glucosinolate and Lipids from Moringa oleifera Lam. Seed. JOTCSA. 2020;7(1):235-44.

DOI: https://doi.org/10.18596/jotcsa.569960.

*Corresponding author. E-mail: atolani.o@unilorin.edu.ng.

\section{INTRODUCTION}

The phytochemicals of plants used in folkloric medicine are apparently responsible for the ethnopharmacological applications. These phytochemicals within plants of same species vary due to differences in their geographical locations. Moringa oleifera Lam. of the monogenetic family, Moringaceae is a widely cultivated medicinal plant with many attributes (1-3). All parts of the plant which include the stem bark, leaves, root, flowers, fruits, pods, and seeds are used in folkloric medicine. Particularly, the seed is used for water purification as flocculants and de-immobiliser of some pathogens (2). Apart from the application of the seed in water purification, the seed is widely consumed by locals in Africa and Asia as a cheap source of anti-hypertension therapy (4). It is also consumed as food or used as food supplement in many part of Africa while its oil is also used for 
cosmeceutical purposes $(5,6)$. Prior to the commencement of this work, there has been paucity of comprehensive detail chemical composition of the seed of $M$. oleifera of Nigerian origin. However, major compounds which include thiocarbamates, isocyanates, glucosinolates, triglyceride, triterpenes and fatty acids have been isolated from the seed majorly of Asian origin (710). Various reports have been documented about the chemical and biological applications of the plant $(4,11,12)$. Despite the numerous emerging reports on $M$. oleifera, the plant is still considered underexplored and underutilised for its many great potential $(2,4)$. Due to environmental impact and geological differences, the phytochemicals of plants often vary from place to place (13). In our previous report (14), we indicated that the ethanolic seed extract of $M$. oleifera of Nigerian origin could trigger infertility of the male through decreased semen $\mathrm{pH}$ and also induce hepatotoxicity due to persistent increase in AST and ALP despite withdrawal of the administration of the extract. In addition, we have also previously shown that the seed has the potential to cause renal toxicity (15). Our previous studies further indicated that though the Moringa oleifera seed extracts had high anti-oxidant potentials, it however showed low proteinase and membrane stabilisation responses (10). In the continuation of our work on the chemical and biological investigations on Moringa oleifera seeds of Nigerian origin $(10,14,16)$, we here report the isolation and characterisation of nine bioactive compounds and additional ten compounds identified with the aid of GC-MS.

\section{MATERIAL AND METHOD}

\section{General Method}

Solvents and chemicals used were analytical grade and where applicable, solvents were re-distilled before use. Infrared spectra were recorded on Shimadzu (8400S) Fourier Transform-Infrared, Shimadzu, Japan, using $\mathrm{KBr}$ pellets. ${ }^{1} \mathrm{H}-\mathrm{NMR}$ (300 $\mathrm{MHz}$ ) and ${ }^{13} \mathrm{C}-\mathrm{NMR}(75 \mathrm{MHz})$ spectra were recorded in deuterated chloroform $\left(\mathrm{CDCl}_{3}\right)$ on Bruker $300 \quad$ Nuclear Magnetic Resonance Spectrometer. Adopting tetramethylsilane (TMS) as an internal standard, all chemical shifts were expressed in parts per million ( $\mathrm{ppm}$ ) downfield from it. Mass spectral data were taken on JMS spectrometer (ESI+) ion trap LCQ and Thermoelectron Corporation. Chromatography was carried out on a silica gel gravity loaded column. Thin layer chromatography (TLC) was performed on pre-coated plates of silica gel 60F-264 (Merck), and spots were visualised using an ultraviolet-light lamp or by exposure to iodine vapour.

\section{GC-FID/GC-MS Analyses}

One of the major oil fraction obtained from the column chromatography was subjected to GC-FID/ GC-MS using an Agilent Technology 7890A gas chromatograph equipped with a HP-5MS column with size $30 \mathrm{~m}$ by $0.32,0.5 \mu \mathrm{m}$ and coupled to a mass spectrometer. The injection and interface were operated at 250 and $380{ }^{\circ} \mathrm{C}$ respectively while the oven temperature was set to operate from 60 to $300{ }^{\circ} \mathrm{C}$ at $5{ }^{\circ} \mathrm{C} \mathrm{min}^{-1}$ and held isothermally at that temperature using a splitless mode at the injection volume of $0.2 \mu \mathrm{L}$. The scan was operated for a total time of $0.5 \mathrm{~h}$ and chemical constituents identified primarily by comparing the fragmentation pattern of each spectrum with reference compounds in the NIST library and literature.

\section{Preparation of Plant Material}

The seed of $M$. oleifera was collected from Northern Nigeria and authenticated at the herbarium of the department of Plant Biology, University of Ilorin, Nigeria. The voucher number UILH/002/1008 was assigned.

\section{Extraction, Column Chromatography Fractionation and Isolation}

The dried, de-husked seed material (2 kg) was pulverised and extracted exhaustively with ethanol for nine days followed by water extraction for 5 days. The aqueous extract was partitioned with dichloromethane (DCM) and the DCM extract was collected and concentrated in vacuo to afford $46.30 \mathrm{~g}$ extract while the ethanolic extract was also concentrated in vacuo using a rotary evaporator to afford $37.10 \mathrm{~g}$ of thick brown syruplike extract. Both the DCM and ethanol extracts were subjected to silica gel column chromatography separately with elution using nhexane, DCM, and methanol in an increasing order of polarity. The DCM extract afforded 23 fractions while the ethanolic extract afforded 42 fractions which were further pulled together based on their TLC profile to afford 7 and 11 major fractions for DCM and ethanol extracts respectively. The major fractions were re-chromatographed and some partially purified compounds were further subjected to preparative thin layer chromatography or washing with solvents as appropriate. Thus, compounds 1-9 were obtained and subjected to various spectroscopic studies for the elucidation of the structures. An oily fraction obtained from the fractionation of the ethanolic extract was subjected to GC-MS analysis.

\section{Extraction, Column Chromatography Fractionation and Isolation}

In order to further identify if any of the compounds have toxic potentials, computational methods were used to predict their toxic effects using ProTox-II web server (17), which has a total 33 models for the prediction of various toxicity endpoints was used for this study, such as acute toxicity, hepatotoxicity, cytotoxicity, carcinogenicity, mutagenicity, immunotoxicity, adverse outcomes pathways and toxicity targets. Additionally, in house based-cytochrome (1A2, 2C9, 2C19, 2D6 and $3 A 4)$ models were used for the prediction. The respective cytochrome models were based on the SuperCyp database (SuperCyp), and were validated both on internal and external datasets. 


\section{RESULTS AND DISCUSSION}

The silica gel column chromatography of the dichloromethane and methanolic extracts of $M$. oleifera seed afforded nine compounds which were characterised using a combination of Nuclear Magnetic Resonance ( $\left.{ }^{1} \mathrm{H}-\mathrm{NMR} /{ }^{13} \mathrm{C}-\mathrm{NMR}\right)$ spectrometry, Electrospray Ionisation Mass Spectrometry (ESI-MS) and Fourier Transform Infrared (FT-IR) spectroscopy as well as in conjunction with literature.

Compound 1: Niazimicin or (O-ethyl-4[a-Lrhamnosyloxy]thiocarbamate). ESI-MS (+) 358 $[\mathrm{M}+\mathrm{H}]^{+}$and $453[\mathrm{M}+26]^{+}$, fragment at $\mathrm{m} / \mathrm{z} 311$ and 253. $\mathrm{m} / \mathrm{z} 357[\mathrm{M}]^{+}$observed for $\mathrm{C}_{16} \mathrm{H}_{23} \mathrm{NO}_{6} \mathrm{~S}$. ${ }^{1} \mathrm{H}-\mathrm{NMR}\left(300 \mathrm{MHz} \mathrm{CDCl}_{3}\right): \delta_{\mathrm{H}}(\mathrm{ppm}) 6.97(\mathrm{H}-2, \mathrm{H}-$ $6, d), 7.27(\mathrm{H}-3, \mathrm{H}-5, \mathrm{~d}), 4.53\left(\mathrm{H}_{2}-7, \mathrm{~d}\right), 5.32(\mathrm{H}-$ $\left.1^{\prime}, \mathrm{d}\right), 3.98\left(\mathrm{H}-2^{\prime}, \mathrm{m}\right), 3.66\left(\mathrm{H}-3^{\prime}, \mathrm{m}\right), 3.23\left(\mathrm{H}-4^{\prime}\right.$, $\mathrm{m}), 3.65\left(\mathrm{H}-5^{\prime}, \mathrm{m}\right), 1.08\left(\mathrm{H}-6^{\prime}, \mathrm{d}\right), 5.01(\mathrm{OH}, \mathrm{d})$, $4.67-4.85(\mathrm{OH}, \mathrm{m}), 4.38(\mathrm{H}, \mathrm{q}), 1.25(\mathrm{H}, \mathrm{t}), 9.85$ (NH). ${ }^{13} \mathrm{C}-\mathrm{NMR}\left[75 \mathrm{MHz},\left(\mathrm{CDCl}_{3}\right)\right]: \delta_{\mathrm{c}} 155.8(\mathrm{C}-1)$, 116.7 (C-2, C-6), 128.4 (C-3), 130.6 (C-4), 131.0 (C-5), $48.6(\mathrm{C}-7), 190.7$ (C-8), $98.2\left(\mathrm{C}-1^{\prime}\right), 70.4$ (C-2'), $70.9\left(\mathrm{C}-3^{\prime}\right), 71.9\left(\mathrm{C}-4^{\prime}\right), 69.4\left(\mathrm{C}-5^{\prime}\right), 17.7$ $\left(\mathrm{C}-6^{\prime}\right), 66.5,14.2\left(\mathrm{OCH}_{2} \mathrm{CH}_{3}\right)$.

Compound 2: Niazidin or (O-cyano-4-(R-Lrhamnosyloxy)benzenethiocarbamate). IR; $U_{\max }$ $\left(\mathrm{cm}^{-1}\right) 3389(\mathrm{NH} / \mathrm{OH}), 3005(\mathrm{Ar} \mathrm{C}-\mathrm{H}), 2924 / 2854$ $(\mathrm{CH}), 2077,1739,1647(\mathrm{~N}-\mathrm{H}$ bend $), 1612(\mathrm{C}=\mathrm{C}$ stretch), $1510(\mathrm{C}=\mathrm{C}), 1465,1420$ (O-H bend), 1379 (C-H bend), 1232 (C-N stretching), 1093 $\left(\mathrm{C}=\mathrm{S}\right.$ stretch). ESI-MS $(+) 355[\mathrm{M}+\mathrm{H}]^{+}, 311$ and 270 fragments. $m / z \quad 355\left[\mathrm{MH}^{+}\right.$observed for $\mathrm{C}_{15} \mathrm{H}_{18} \mathrm{~N}_{2} \mathrm{O}_{6} \mathrm{~S}$. ${ }^{1} \mathrm{H}$ NMR (300 $\left.\mathrm{MHz} \mathrm{CDCl}_{3}\right): \delta_{\mathrm{H}}(\mathrm{ppm})$ $6.95(\mathrm{H}-2, \mathrm{H}-6, d, \mathrm{~J}=8.4 \mathrm{~Hz}), 7.19(\mathrm{H}-3, \mathrm{H}-5, \mathrm{~d}, \mathrm{~J}$ $=8.4 \mathrm{~Hz}), 4.58(\mathrm{H}-7, \mathrm{~d}), 5.32\left(\mathrm{H}-1^{\prime}, \mathrm{d}\right), 3.82\left(\mathrm{H}-2^{\prime}\right.$ d), $3.66\left(\mathrm{H}-3^{\prime}, \mathrm{m}\right), 3.23\left(\mathrm{H}-4^{\prime}, \mathrm{s}\right), 3.65\left(\mathrm{H}-5^{\prime}, \mathrm{m}\right)$, $1.00\left(\mathrm{H}-6^{\prime}, \mathrm{d}\right), 4.70-5.01(\mathrm{OH}, \mathrm{m}), 7.80(\mathrm{NH})$. ${ }^{13} \mathrm{C}-\mathrm{NMR}$ [75 MHz, $\left.\left(\mathrm{CDCl}_{3}\right)\right]$ : $\delta_{\mathrm{C}} 156.5(\mathrm{C}-1), 117.3$ (C-2, C-6), 129.2 (C-3), 130.1 (C-4), 129.7 (C-5), 179.1 (C-8), $98.5\left(\mathrm{C}-1^{\prime}\right), 70.2\left(\mathrm{C}-2^{\prime}\right), 70.4$ (C-3'), $70.8\left(\mathrm{C}-4^{\prime}\right), 69.3\left(\mathrm{C}-5^{\prime}\right), 17.7\left(\mathrm{C}-6^{\prime}\right)$.

Compound 3: Glucomoringin isothiocyanate or 4(a-L-rhamnosyloxy)benzyl isothiocyanate. IR; U max $\left(\mathrm{cm}^{-1}\right) 3358(\mathrm{OH}), 2928 / 2854(\mathrm{CH}), 2210(\mathrm{CN})$, 2065, 1743, 1654 (N-H bend), 1612 ( $\mathrm{C}=\mathrm{C}$ stretch), 1510,1450 ( $\mathrm{C}=\mathrm{C}), 1388$ ( $\mathrm{C}-\mathrm{H}$ bend), 1230 (C-N stretching), 1093 ( $\mathrm{C}=\mathrm{S}$ stretch). ESI$\mathrm{MS}(+) 312[\mathrm{M}+\mathrm{H}]^{+}, 311,270$ and 253 fragments. $\mathrm{m} / \mathrm{z} 310.5[\mathrm{M}]^{+}$observed for $\mathrm{C}_{14} \mathrm{H}_{17} \mathrm{O}_{5} \mathrm{NS}$. ${ }^{1} \mathrm{H} \mathrm{NMR}$ (300 $\left.\mathrm{MHz}_{2} \mathrm{CDCl}_{3}\right): \delta_{\mathrm{H}}(\mathrm{ppm}) 7.21(\mathrm{H}-2, \mathrm{H}-6, d)$, $7.02(\mathrm{H}-3, \mathrm{H}-5, \mathrm{~d}, \mathrm{~J}=9.3 \mathrm{~Hz}), 4.62\left(\mathrm{H}_{2}-7, \mathrm{~s}\right), 5.45$ $\left(\mathrm{H}-1^{\prime}, \mathrm{d} \mathrm{J}=1.2\right), 4.10\left(\mathrm{H}-2^{\prime} \mathrm{br}, \mathrm{s}\right), 3.99\left(\mathrm{H}-3^{\prime}, \mathrm{dd}\right.$, $\mathrm{J}=6 \mathrm{~Hz}), 3.56\left(\mathrm{H}-4^{\prime}, \mathrm{s}\right), 3.79\left(\mathrm{H}-5^{\prime}, \mathrm{m}\right), 1.26(\mathrm{H}-$ $\left.6^{\prime}, d, j=5.4 \mathrm{~Hz}\right), 4.81(\mathrm{OH}, \mathrm{s}), 2.63(2 \mathrm{OH}, \mathrm{br} \mathrm{s})$, $7.80(\mathrm{NH}) .{ }^{13} \mathrm{C}-\mathrm{NMR}\left[75 \mathrm{MHz},\left(\mathrm{CDCl}_{3}\right)\right]: \delta_{c} 156.1$ (C-1), 116.7 (C-2), 129.3 (C-3), 129.0 (C-4), 129.1 (C-5), 116.6 (C-6), 48.8 (C-7), 130.9 (C-8), $98.2\left(\mathrm{C}-1^{\prime}\right), 70.7\left(\mathrm{C}-2^{\prime}\right), 70.3\left(\mathrm{C}-3^{\prime}\right), 72.7\left(\mathrm{C}-4^{\prime}\right)$, $69.1\left(\mathrm{C}-5^{\prime}\right), 17.4\left(\mathrm{C}-6^{\prime}\right)$.

Compound 4: Niazinin acetate or 4-(3'-O-acetyl-aL-rhamnosyloxy)benzyl isothiocyanate. ESI-MS (+)
$432[\mathrm{MH}+\mathrm{Na}+\mathrm{Na}]^{+}, 310$ and 270 fragments. $\mathrm{m} / \mathrm{z}$ $432.02\left[\mathrm{M}+\mathrm{NH}_{4}\right]^{+}$observed for $\mathrm{M}^{+} \mathrm{C}_{17} \mathrm{H}_{23} \mathrm{NO}_{7} \mathrm{~S} .{ }^{1} \mathrm{H}$ NMR $\left(300 \mathrm{MHz}_{2} \mathrm{CDCl}_{3}\right): \delta_{\mathrm{H}}(\mathrm{ppm}) 7.03(\mathrm{H}-2, \mathrm{H}-6$, d), $7.18(\mathrm{H}-3, \mathrm{H}-5, d), 4.63\left(\mathrm{H}_{2}-7, \mathrm{~d}\right), 5.543\left(\mathrm{H}-1^{\prime}\right.$, d), $4.10\left(\mathrm{H}-2^{\prime} \mathrm{br}, \mathrm{s}\right), 4.07\left(\mathrm{H}-3^{\prime}, \mathrm{d}\right), 4.87\left(\mathrm{H}-4^{\prime}, \mathrm{t}\right)$, $3.96\left(\mathrm{H}-5^{\prime}, \mathrm{m}\right), 1.01\left(\mathrm{H}-6^{\prime}, \mathrm{d}\right), 3.77,3.24\left(2^{\prime}-\mathrm{OH}\right.$, $\left.4^{\prime}-\mathrm{OH}, \mathrm{s}\right), 7.60(\mathrm{NH}, \mathrm{t}), 2.19(\mathrm{OAc}, \mathrm{br}, \mathrm{s})$.

Compound 5: Niazinin triacetate or 4-(2',3',4'-Otriacetyl-a-L-rhamnosyloxy)benzyl isothiocyanate. IR; U max $\left(\mathrm{cm}^{-1}\right) 3381$ (UN-H/O-H), 2926/2854 (UC-H), 2171 (UCN), 2088, 1741 (UC=0), 1654 (UC-N bend), 1612 (UC=C stretch), 1510, 1458 (UC=C), 1379 (UC-H bend), 1236 (UC-N stretch), 1093 (UC=S stretch). ESI-MS (+) $487\left[\mathrm{M}+\mathrm{NH}_{4}\right]^{+}, 325,310$ and 270 fragments. $m / z$ 487.15 $\left[\mathrm{M}+\mathrm{NH}_{4}\right]^{+}$observed for $\mathrm{C}_{21} \mathrm{H}_{27} \mathrm{NO}_{9} \mathrm{~S} .{ }^{1} \mathrm{H}$ NMR (300 $\left.\mathrm{MHz}_{1} \mathrm{CDCl}_{3}\right): \delta_{\mathrm{H}}(\mathrm{ppm})$ $7.00(\mathrm{H}-2, \mathrm{H}-6, d, \mathrm{~J}=8.4 \mathrm{~Hz}), 7.12(\mathrm{H}-3, \mathrm{H}-5, d, \mathrm{~J}$ $=8.4 \mathrm{~Hz}), 4.58\left(\mathrm{H}_{2}-7, \mathrm{~d}, \mathrm{~J}=6.9 \mathrm{~Hz}\right), 5.56\left(\mathrm{H}-1^{\prime}\right.$, d), $5.32\left(\mathrm{H}-2^{\prime} \mathrm{br}, \mathrm{dd}, \mathrm{J}=5.1 \mathrm{~Hz}\right), 5.21\left(\mathrm{H}-3^{\prime}, \mathrm{dd}\right)$, $4.99\left(\mathrm{H}-4^{\prime}, \mathrm{t}\right), 3.93\left(\mathrm{H}-5^{\prime}, \mathrm{m}\right), 1.01\left(\mathrm{H}-6^{\prime}, \mathrm{d}, \mathrm{J}=\right.$ $6.9 \mathrm{~Hz}), 3.45(\mathrm{OMe}, \mathrm{s}), 4.62(\mathrm{NH}, \mathrm{t}), 1.99$ (OAc, $\mathrm{br}, \mathrm{s}) .{ }^{13} \mathrm{C}-\mathrm{NMR}\left[75 \mathrm{MHz},\left(\mathrm{CDCl}_{3}\right)\right]: \delta_{\mathrm{C}} 156.2(\mathrm{C}-1)$, $116.7(\mathrm{C}-2), 128.4(\mathrm{C}-3), 131.0(\mathrm{C}-4), 128.2 .1$ (C5), 116.9 (C-6), 48.3 (C-7), 190.7 (C-8), 97.8 (C$\left.1^{\prime}\right), 76.8\left(\mathrm{C}-2^{\prime}\right), 72.5\left(\mathrm{C}-3^{\prime}\right), 71.5\left(\mathrm{C}-4^{\prime}\right), 71.0(\mathrm{C}-$ $\left.5^{\prime}\right), 17.7$ (C-6'), 174.2, 174.0, 173.7 (3C=0), $22.8,21.2$ and 19.9 (3OAc).

Compound 6: Niazirinin. 4-[(4'-O-acetyl-a-Lrhamnosyloxy)benzyl] nitrile. ESI-MS $(+) m / z$ $357.89\left[\mathrm{M}+\mathrm{NH}_{4}+\mathrm{NH}_{4}\right]^{+}$was obtained for $\mathrm{C}_{16} \mathrm{H}_{19} \mathrm{NO}_{6} .{ }^{1} \mathrm{H} \mathrm{NMR}\left(300 \mathrm{MHz} \mathrm{CDCl}_{3}\right): \delta_{\mathrm{H}} 7.07(\mathrm{H}-$ 2, H-6, d, J = 9.0 Hz), $7.16(\mathrm{H}-3, \mathrm{H}-5, d, \mathrm{~J}=9.0$ $\mathrm{Hz}), 3.68\left(\mathrm{H}_{2}-7, \mathrm{~s}\right), 5.38\left(\mathrm{H}-1^{\prime}, \mathrm{d}, \mathrm{J}=2.4\right), 4.15$ $\left(\mathrm{H}-2^{\prime} \mathrm{m}\right), 4.09\left(\mathrm{H}-3^{\prime}, \mathrm{dd}\right), 4.62\left(\mathrm{H}-4{ }^{\prime}, \mathrm{t}\right), 3.58(\mathrm{H}-$ $\left.5^{\prime}, \mathrm{m}, \mathrm{J}=9.3\right), 1.19\left(\mathrm{H}-6^{\prime}, \mathrm{d}, \mathrm{J}=7.2 \mathrm{~Hz}\right), 2.00$ $(2 \mathrm{OH}, \mathrm{br}, \mathrm{s}), 2.04$ (OCOMe, s), $4.62(\mathrm{NH}, \mathrm{t}), 1.99$

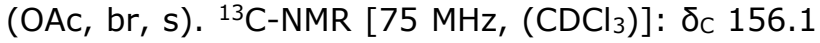
$(\mathrm{C}-1), 116.7$ (C-2), 129.3 (C-3), 131.2 (C-4), 129.7 (C-5), 116.9 (C-6), 22.9 (C-7), 123.8 (C-8), $98.0\left(\mathrm{C}-1^{\prime}\right), 71.3\left(\mathrm{C}-2^{\prime}\right), 70.8\left(\mathrm{C}-3^{\prime}\right), 72.5\left(\mathrm{C}-4^{\prime}\right)$, $66.7\left(\mathrm{C}-5^{\prime}\right), 17.6\left(\mathrm{C}-6^{\prime}\right), 173.9,\left(\mathrm{OCOCH}_{3}\right), 21.1$ $\left(\mathrm{OCOCH}_{3}\right)$.

Compound 7: Glucotropaeolin or (glucosinolate). IR; $U_{\max }\left(\mathrm{cm}^{-1}\right) 3412$ (Uо-H), 2931 (UC-H), 1645 (UC=N stretch), 1610 (UC=C stretch), 1510 (UC-H), 1384 (Us=o stretch), 1254 (Uc-O-C/C-N stretch), 1234 (Uc-N stretch). ESI-MS (+) $\mathrm{m} / \mathrm{z} 425.11[\mathrm{M}+\mathrm{H}]^{+}$was obtained for $\mathrm{C}_{14} \mathrm{H}_{18} \mathrm{NO}_{10} \mathrm{~S}_{2}{ }^{-}$. ${ }^{1} \mathrm{H}$ NMR $(300 \mathrm{MHz}$, $\left.\mathrm{CDCl}_{3}\right): \delta_{\mathrm{H}}(\mathrm{ppm}) 3.30-3.66\left(\mathrm{~m}, 4 \mathrm{H}, \mathrm{H}-2^{\prime}, \mathrm{H}-3^{\prime}, \mathrm{H}-\right.$ $\left.4^{\prime}, \mathrm{H}-5^{\prime}\right), 3.67-3.73\left(\mathrm{~m}, 2 \mathrm{H}, \mathrm{H}-6 \mathrm{a}^{\prime}, \mathrm{H}-6 \mathrm{~b}^{\prime}\right), 2.00$ $\left(3 \mathrm{OH}^{\prime}, \mathrm{br}, \mathrm{s}\right), 4.07\left(\mathrm{~s}, 2 \mathrm{H}, \mathrm{CH}_{2} \mathrm{Ph}\right), 4.77(\mathrm{~d}, 1 \mathrm{H}, \mathrm{H}-$ $\left.1^{\prime}\right), 5.33(\mathrm{~s}, \mathrm{br}$,

ArOH), 7.18 (dd, ArH-4, 8, J = 8.4 Hz), 7.02 (dd, ArH-5, 7, J = 8.4 Hz).

Compound 8: Triolein. IR; $U_{\max }\left(\mathrm{cm}^{-1}\right)$ 2926/2854 (UC-H), 1747 (UC=O), 1610 (UC=C stretch), 1456 (UC=N stretch), 1238 (UC-O-C stretch). ESI-MS (+) $\mathrm{m} / z 903.47\left[\mathrm{M}+\mathrm{NH}_{4}\right]^{+}$obtained for $\mathrm{C}_{57} \mathrm{H}_{104} \mathrm{O}_{6} .{ }^{1} \mathrm{H}-$ NMR $\left(300 \mathrm{MHz}, \mathrm{CDCl}_{3}\right): \delta_{\mathrm{H}}(\mathrm{ppm})$ 0.88-0.90 (t, $\left.\mathrm{CH}_{3}\right), 1.25-1.30,1.60\left(\mathrm{~m},-\mathrm{CH}_{2}-\right), 1.97(\mathrm{~m}$, $\left.\mathrm{CH}_{2} \mathrm{C}=\mathrm{C}-\right), 5.39$ (m, $-\mathrm{CH}$ of olefins) $5.24(\mathrm{~m}, \mathrm{CH}$ 
of glycerol), 4.10-4.32 (ddd, $\mathrm{CH}_{2}$ of glycerol). ${ }^{13} \mathrm{C}-\quad \mathrm{NMR}\left[75 \mathrm{MHz},\left(\mathrm{CDCl}_{3}\right)\right]: \delta_{\mathrm{c}}(\mathrm{ppm}) 173.3(\mathrm{C}=\mathrm{O}, \mathrm{C}-$

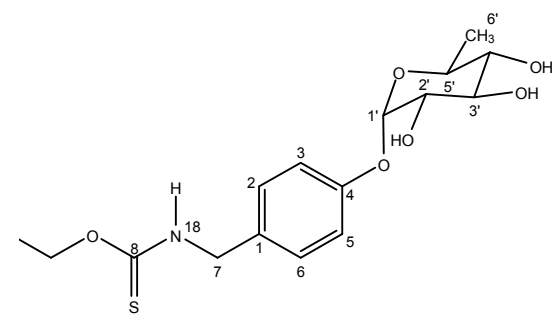

1

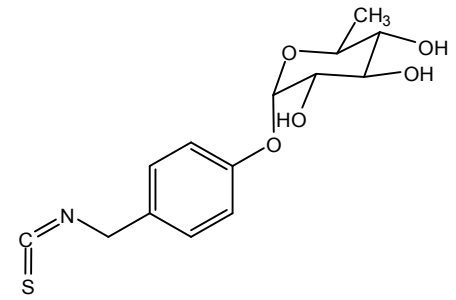

3<smiles>COC(=O)NCc1ccc(OC2OC3CC(C)C(O)C(O3)O2)cc1</smiles>

5<smiles>CC1OC(Oc2ccc(CNC(=S)OC#N)cc2)C(O)C(O)C(O)C1O</smiles>

2<smiles>COC(=O)C1(C)OC(Oc2ccc(CNC(=S)OC)cc2)C(O)C(O)C1O</smiles>

4<smiles>CCC1OC(Oc2ccc(CC#N)cc2)C(O)C(O)C1OC(=O)O</smiles>

6<smiles>O=C(N=C(Cc1ccc(O)cc1)SC1(O)OC2(CO)C(O)C(O)C1(CO)C2O)O[Na]</smiles>

7

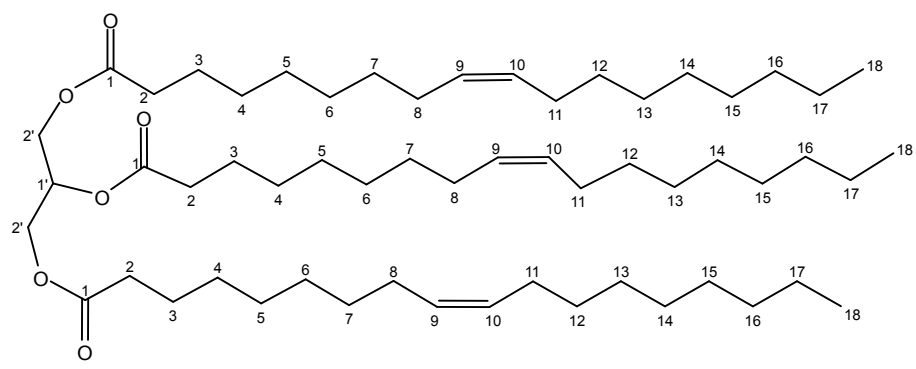

8

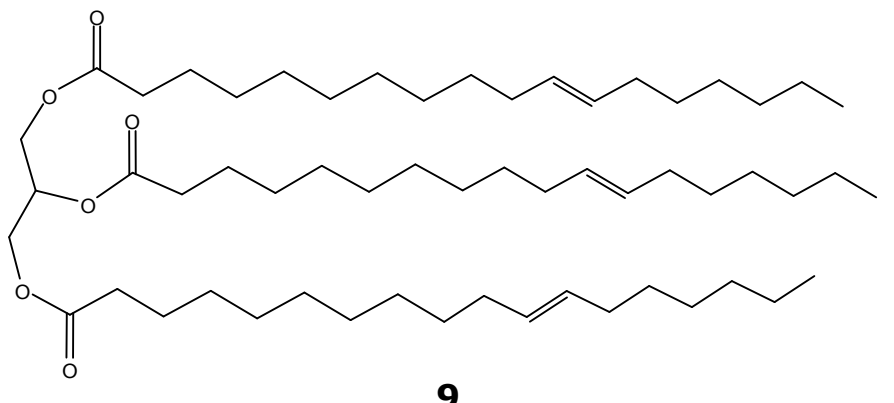

9

Figure 1: Strutures of Niazimicin 1, Niazidin 2, Glucomoringin isothiocyanate 3, Niazinin acetate 4, Niazinin triacetate 5, Niazirinin 6, Glucotropaeolin 7, Triolein 8, Trivaccenin $\mathbf{9}$. 
1), 34.3 (C-2), 25.0 (C-3), 29.1 (C-4), 29.9 (C-5), 29.8 (C-6), 32.0 (C-7), 29.6 (C-8), 129.9 (C-9), 129.8 (C-10), $29.5(\mathrm{C}-11), 31.9(\mathrm{C}-12), 29.4$ (C13), 29.3 (C-14), 29.3 (C-15), 32.0 (C-16), 22.8 (C-17), 14.2 (C-18), 69.1 (C-1' of glycerol backbone), 62.2 (O-C-2' of glycerol backbone).

Compound 9: Trivaccenin. IR; $U_{\max }\left(\mathrm{cm}^{-1}\right)$ 2926/2854 ( $\left.U_{\mathrm{C}-\mathrm{H}}\right), 1747\left(\mathrm{U}_{\mathrm{C}=\mathrm{O}}\right), 1612$ (U $\mathrm{U}_{\mathrm{C}=\mathrm{C}}$ stretch), 1465 ( $\mathrm{U}_{\mathrm{C}=\mathrm{N}}$ stretch), 1238 (U $\mathrm{U}_{\mathrm{C}-\mathrm{O}-\mathrm{C}}$ stretch). ESI-MS (+) $m / z 903.47\left[\mathrm{M}+\mathrm{NH}_{4}\right]^{+}$observed for $\mathrm{C}_{57} \mathrm{H}_{104} \mathrm{O}_{6}$. $m / z 824$ observed for $\left[\mathrm{M}^{+}-\mathrm{C}_{9} \mathrm{H}_{18}\right]^{+}$fragment. ${ }^{1} \mathrm{H}-$ NMR (300 MHz, $\left.\mathrm{CDCl}_{3}\right): \delta_{\mathrm{H}}(\mathrm{ppm})$ 0.88-0.90 (t, $\left.\mathrm{CH}_{3}\right), 1.25-1.30,1.61\left(\mathrm{~m},-\mathrm{CH}_{2}^{-}\right), 1.98(\mathrm{~m},-$ $\left.\mathrm{CH}_{2} \mathrm{C}=\mathrm{C}-\right), 5.39$ (m, $-\mathrm{CH}$ of olefins), 5.25 (m, $\mathrm{CH}$ of glycerol), $4.10-4.32$ (ddd, $\mathrm{CH}_{2}$ of glycerol). ${ }^{13} \mathrm{C}$ NMR $\left[75 \mathrm{MHz},\left(\mathrm{CDCl}_{3}\right)\right]: \delta_{\mathrm{c}}(\mathrm{ppm}) 173.3(\mathrm{C}=\mathrm{O})$, 34.1 (C-2), 25.0 (C-3), 29.1 (C-4), 29.2 (C-5), 29.3 (C-6), 29.4 (C-7), 29.7 (C-8), 29.8 (C-9), 31.9 (C-10), 129.9 (C-11), $129.8(\mathrm{C}-12), 31.9$ (C13), 29.8 (C-14), 29.7 (C-15), 29.4 (C-16), 22.8 $(\mathrm{C}-17), 14.2(\mathrm{C}-18), 69.0\left(\mathrm{C}-1^{\prime}\right.$ of glycerol backbone), 62.2 (O-C-2' of glycerol backbone).

Composition of the Fraction: The major oily fraction obtained from the silica gel fractionation of the ethanolic extract was subjected to GC-FID/GCMS and FT-IR analyses. Ten compounds, primarily fatty acids/esters, were identified (Table 1). The major compound was oleic acid (45.19\%), followed by 13 -docosenoic acid $(16.15 \%)$, stearic acid $(10.56 \%)$, palmitic acid $(9.21 \%)$ and methyl transvaccenate $(6.48 \%)$. Other compounds which were obtained in low yield includes methyl palmitate $(2.48 \%)$, a-monopalmitin $(2.12 \%)$, methyl stearate $(1.32 \%)$. Two non-fatty acids/esters, p- hydroxybenzyl cyanide (4.16\%) and a -Irhamnopyranose $(2.33 \%)$ were however obtained in minute quantities. The FT-IR spectrum of the fraction indicated major peaks at 3392, 2926, 2854, 2173, 2090, 1741, 1612, 1510, 1386, 1236, 1021,983 and $669 \mathrm{~cm}^{-1}$.

From the in silico studies, compounds which include niazidin, niazinin acetate, and niazinin triacetate were estimated to possess high immunotoxicity (as shown in Table 2). Also, most of the compounds were predicted as cytochrome 3A4 substrate and/or inhibitors with good probability. Additionally, triolein and trivaccenin were predicted to be carcinogenic and mildly mutagenic. Triolein and trivaccenin were predicted with good pharmacophore fit values to justify probable binding with amine oxidase.

Sixteen carbon signals were depicted by the $\mathrm{C}-13$ spectrum of the compound $\mathbf{1}$ with the characteristic $\mathrm{C}=\mathrm{S}$ signal appearing at $190 \mathrm{ppm}$. The C-O carbon of the aromatic was observed at $155 \mathrm{ppm}$ while the aromatic $\mathrm{C}=\mathrm{C}$ bonds were clearly depicted 116, 128, 130 and $131 \mathrm{ppm}$. The aromatic carbon signals were corroborated by the proton NMR signals between 6.97 and 7.27 ppm. The fragment ion at 311 resulting from the loss of the ethoxy group of from the niazimicin in the ESIMS spectrum strengthens the claim about the structure of the elucidated compound as O-ethyl4[a-L-rhamnosyloxy] thiocarbamate with molecular formula $\mathrm{C}_{16} \mathrm{H}_{23} \mathrm{NO}_{6} \mathrm{~S}$. The spectra data of the niazimicin was confirmed by comparing with literatures $(7,18-20)$. The proton and carbon 13 NMR of niazidin $\mathbf{2}$ ran in deuterated chloroform was carefully compared to the literature (8).

Table 1: Compounds identified in a major oily fraction of the ethanolic extract.

\begin{tabular}{llllll}
\hline SN & RT (min) & Compounds & MF & \% Composition & $\begin{array}{c}\text { Important } \\
\text { Fragments }\end{array}$ \\
\hline 1 & 6.93 & a -l-rhamnopyranose & $\mathrm{C}_{6} \mathrm{H}_{12} \mathrm{O}_{5}$ & 2.33 & $43,73,128$ \\
2 & 12.41 & p-Hydroxybenzyl cyanide & $\mathrm{C}_{8} \mathrm{H}_{7} \mathrm{NO}$ & 4.16 & $51,78,133$ \\
3 & 17.24 & Methyl palmitate & $\mathrm{C}_{17} \mathrm{H}_{34} \mathrm{O}_{2}$ & 2.48 & $74,87,270$ \\
4 & 18.65 & Palmitic acid & $\mathrm{C}_{16} \mathrm{H}_{32} \mathrm{O}_{2}$ & 9.21 & $43,73,256$ \\
5 & 20.27 & Methyl trans-vaccenate & $\mathrm{C}_{19} \mathrm{H}_{36} \mathrm{O}_{2}$ & 6.48 & $55,69,296$ \\
6 & 20.59 & Methyl stearate & $\mathrm{C}_{19} \mathrm{H}_{38} \mathrm{O}_{2}$ & 1.32 & $74,87,298$ \\
7 & 21.41 & Oleic acid & $\mathrm{C}_{18} \mathrm{H}_{34} \mathrm{O}_{2}$ & 45.19 & $41,55,280$ \\
8 & 21.60 & Stearic acid & $\mathrm{C}_{18} \mathrm{H}_{36} \mathrm{O}_{2}$ & 10.56 & $43,55,284$ \\
9 & 25.22 & a-Monopalmitin & $\mathrm{C}_{19} \mathrm{H}_{38} \mathrm{O}_{4}$ & 2.12 & $43,57,313$ \\
\hline 10 & 27.10 & $13-$-Docosenoic acid & $\mathrm{C}_{22} \mathrm{H}_{42} \mathrm{O}_{2}$ & 16.15 & $41,55,325$ \\
\hline
\end{tabular}

RT: Retention Time; MF: Molecular Formula 
Table 2: Computational toxicity evaluation result from the ProTox-II platform.

\begin{tabular}{|c|c|c|c|c|c|c|c|c|}
\hline $\begin{array}{l}\text { Compound } \\
\text { name }\end{array}$ & $\begin{array}{l}\text { Pubchem } \\
\text { ID }\end{array}$ & $\begin{array}{l}\text { Predicted } \\
\text { LD50 value } \\
\text { and Tox class }\end{array}$ & $\begin{array}{l}\text { Prediction } \\
\text { accuracy } \\
(\%)\end{array}$ & $\begin{array}{l}\text { Toxicity } \\
\text { targets }\end{array}$ & $\begin{array}{l}\text { Average } \\
\text { pharmacophore } \\
\text { fits (\%) }\end{array}$ & $\begin{array}{l}\text { Toxicity end } \\
\text { points }\end{array}$ & $\begin{array}{l}\text { Prediction } \\
\text { probability }\end{array}$ & Cytochrome prediction \\
\hline Niazimicin & 5471459 & $\begin{array}{l}3750(\mathrm{mg} / \mathrm{kg}) \text {, } \\
\text { Tox class: } 5\end{array}$ & 68.07 & - & - & Immunotoxicity & 0.62 & CYP3A4 substrate $(60 \%)$ \\
\hline Niazidin & 11792427 & $\begin{array}{l}3750(\mathrm{mg} / \mathrm{kg}) \text {, } \\
\text { Tox class: } 5\end{array}$ & 68.07 & - & - & Immunotoxicity & 0.84 & $\begin{array}{l}\text { CYP3A4 substrate/inhibitor } \\
(60 \%)\end{array}$ \\
\hline $\begin{array}{l}\text { Glucomoringin } \\
\text { isothlocyanate }\end{array}$ & 153557 & $\begin{array}{l}3750(\mathrm{mg} / \mathrm{kg}) \text {, } \\
\text { Tox class: } 5\end{array}$ & 69.06 & - & - & Immunotoxicity & 0.64 & CYP3A4 substrate $(59 \%)$ \\
\hline \multirow[t]{2}{*}{ Nlazinin acetate } & - & $\begin{array}{l}3750(\mathrm{mg} / \mathrm{kg}) \text {, } \\
\text { Tox class: } 5\end{array}$ & 69.26 & Amine Oxidase & 31.28 & Immunotoxicity & 0.98 & CYP3A4 substrate (67\%) \\
\hline & & & & $\begin{array}{l}\text { Prostaglandin } \\
\text { G/H synthase } 1\end{array}$ & 42.44 & & & \\
\hline \multirow[t]{2}{*}{$\begin{array}{l}\text { Nlazinin } \\
\text { triacetate }\end{array}$} & - & $\begin{array}{l}3750(\mathrm{mg} / \mathrm{kg}) \text {, } \\
\text { Tox class: } 5\end{array}$ & 69.26 & Amine Oxidase & 31.28 & Immunotoxicity & 0.98 & $\begin{array}{l}\text { CYP3A4 substrate, } \\
\text { CYP3A4 inhibitor, } \\
\text { CYP2C19 inhibitor }(68 \%)\end{array}$ \\
\hline & & & & $\begin{array}{l}\text { Prostaglandin } \\
\text { G/H synthase } 1\end{array}$ & 42.44 & & & \\
\hline Nlazirinin & 10426197 & $\begin{array}{l}4000(\mathrm{mg} / \mathrm{kg}) \text {, } \\
\text { Tox class: } 5\end{array}$ & 70.97 & - & - & - & - & CYP3A4 substrate $(59 \%)$ \\
\hline Glucotropaeolin & 656498 & $\begin{array}{l}2000(\mathrm{mg} / \mathrm{kg}) \text {, } \\
\text { Tox class: } 4\end{array}$ & 23.00 & - & - & - & - & CYP3A4 substrate ( $52 \%)$ \\
\hline \multirow[t]{2}{*}{ Triolein } & 5497163 & $\begin{array}{l}3520(\mathrm{mg} / \mathrm{kg}) \text {, } \\
\text { Tox class: } 5\end{array}$ & 72.90 & Amine Oxidase & 63.88 & Carcinogenicity & 0.70 & - \\
\hline & & & & $\begin{array}{l}\text { Prostaglandin } \\
\text { G/H synthase } 1\end{array}$ & 58.45 & Mutagenecity & 0.57 & \\
\hline \multirow[t]{2}{*}{ Trivaccenin } & 14029829 & $\begin{array}{l}3520(\mathrm{mg} / \mathrm{kg}) \text {, } \\
\text { Tox class: } 5\end{array}$ & 72.90 & Amine Oxidase & 63.88 & Carcinogenicity & 0.70 & - \\
\hline & & & & $\begin{array}{l}\text { Prostaglandin } \\
\text { G/H synthase } 1\end{array}$ & 58.45 & Mutagenecity & 0.57 & \\
\hline
\end{tabular}

NB: Compounds marked in red have higher toxicity potential.

The sugar moiety was confirmed following the appearance of the proton doublet at 5.32, 3.82, $3.66,3.23,3.65,1.00$ and broad hydroxyl proton signals at $4.70-5.01 \mathrm{ppm}$. The anomeric $\left(\mathrm{H}-1^{\prime}\right)$ proton signal indicates that the sugar moiety is linked with the aglycone by a a-glycosidic bond suggesting an a-L-rhamonoside (8, 18). The agylcone moiety had protons which mutually coupled at $7.19(\mathrm{~J}=8.4 \mathrm{~Hz})$ and $6.95(\mathrm{~J}=8.4 \mathrm{~Hz})$ supporting a para-substituted benzene structure. The evidence of the para-disubstituted benzene was also affirmed by the characteristic stretching vibration at $835 \mathrm{~cm}^{-1}$ in the infrared spectrum. The $\mathrm{N}-\mathrm{H}$ bond linked to benzylic methylene was characteristically supported by the peaks at $m / z$ 253, 270 and 311 corresponding to the fragment ions at $\left[\left(\mathrm{M}^{+}+1\right)-\mathrm{C}_{2} \mathrm{H}_{2} \mathrm{~N}_{2} \mathrm{OS}\right],\left[\left(\mathrm{M}^{+}+1\right)-\right.$ $\left.\mathrm{C}_{2} \mathrm{HN}_{2} \mathrm{~S}\right]$ and $\left[\left(\mathrm{M}^{+}-\mathrm{HOCN}\right]\right.$ respectively.

In the infrared spectrum, the overlap of the $\mathrm{N}-\mathrm{H}$ and $\mathrm{O}-\mathrm{H}$ stretching vibration was observed at 3389 $\mathrm{cm}^{-1}$ while the $\mathrm{C}-\mathrm{H}$ bond stretching of the methyl group appeared at $2962 \mathrm{~cm}^{-1}$ with a corresponding bending vibration at $1379 \mathrm{~cm}^{-1}$. The asymmetric stretching and symmetrical stretching vibrations of the methylene appeared at 2924 and $2854 \mathrm{~cm}^{-1}$.

The $\mathrm{O}-\mathrm{H}$ bending appeared at $1420 \mathrm{~cm}^{-1}$. The characteristic cyanate (R-O-CN) and C-S vibrations appeared at 2077 and $671 \mathrm{~cm}^{-1}$ respectively. Thus, the structure of the compound $\mathbf{2}$ was unambiguously established as O-cyano-4-(R-Lrhamnosyloxy)benzenethiocarbamate otherwise known as niazidin (Fig. 1).

In order to confirm the structure of the compound 3, the spectra data were compared with those reported in the literature $(8,18,20,21)$. The ${ }^{1} \mathrm{H}$ NMR spectrum indicated two aromatic protons resonating at $\delta 7.21$ and 7.02 with coupling constant of $9.3 \mathrm{~Hz}$ suggesting a para-substituted benzene structure. The absence of the $\mathrm{N}-\mathrm{H}$ chemical shift in the ${ }^{1} \mathrm{H}-\mathrm{NMR}$ spectrum supports the isothiocyanate nature of the compound. The anomeric proton at $\delta 5.45$ confirms the glycosidic nature which was further strengthened by the carbon signal at $\delta 98.2$. The bands at 3358, 2210 and $1230 \mathrm{~cm}^{-1}$ corresponding to the hydroxyl, nitrile $(\mathrm{CN})$ and $\mathrm{C}-\mathrm{N}$ stretching vibration in the infrared spectrum corroborate this position. The mass spectrum exhibited the molecular ion at $\mathrm{m} / \mathrm{z}$ 310.5 corresponding to $\mathrm{C}_{14} \mathrm{H}_{17} \mathrm{O}_{5} \mathrm{NS}$. Thus, the combination of the mass spectra data and NMR data with the infrared report unequivocally assisted the identification of the compound as 4-(a-Lrhamnosyloxy)benzyl isothiocyanate otherwise called glucomoringin isothiocyanate. Many isothiocyanates and glucosinolates have been reportedly isolated from various plants (22).

Compound 4 was identified principally using the ESI-MS and $1 \mathrm{H}-\mathrm{NMR}$ data and comparing with the literature $(8,23,24)$. The molecular weight of the compound was obtained as a disodiated species at $m / z$ 432.02. The compound was identified as 4-(3'O-acetyl-a-L-rhamnosyloxy)benzyl isothiocyanate also called niazinin acetate 4 . The compound has been isolated as a minor constituent of the seed of M. oleifera previously (24).

Compound $\mathbf{5}$ has structural similarity with niazinin. However, when the ${ }^{1} \mathrm{H}-\mathrm{NMR}$ data was compared with that of niazinin $(18,23,25)$, the broad $\mathrm{OH}$ peak appearing between 4.8-5.1 ppm was absent, implying that the hydroxyl groups have been acetylated. In addition, the molecular ion adduct peak at $m / z \quad 487.15$ in the mass spectrum in 
conjunction with the three carbonyl carbon signals (at $\delta 174.2,174.0$ and 173.7) and three addition signals at 22.8, 21.2 and 19.9 for methyl carbons of the acetyl in the ${ }^{13} \mathrm{C}-\mathrm{NMR}$ attested to the triacetate nature of the compound. The $\delta 190.7$ signal confirms the presence of the CS carbon while the isothiocyanate band was confirmed at $2171 \mathrm{~cm}^{-1}$ in the infrared data. The $\mathrm{N}-\mathrm{H}$ stretching vibration was slightly reduced and observed at $3381 \mathrm{~cm}^{-1}$ due to intra-molecular hydrogen bonding. The study of the combined spectral data and comparison with literature data facilitated the establishment of the compounds as $4-\left(2^{\prime}, 3^{\prime}, 4^{\prime}-0-\right.$ triacetyl-a-L-rhamnosyloxy)benzyl isothiocyanate otherwise referred to as niazinin triacetate an analogue of and niazinin and 4-(3'-O-acetyl-a-Lrhamnosyloxy)benzyl isothiocyanate previously isolated from the seed of $M$. oleifera $(18,19)$. This is the first account of a fully acetylated niazinin (with three acetyl group) from Moringa oleifera seed.

The structure of the compound $\mathbf{6}$ was elucidated using the ${ }^{1} \mathrm{H}-\mathrm{NMR},{ }^{13} \mathrm{C}-\mathrm{NMR}$ and ESI-MS data and further confirmed by comparing the data to that reported for niazirinin (7). The molecular ion adduct peak at $m / z 357.89$ in the ESI mass spectrum in conjunction with the carbonyl carbon signals (at $\delta$ 173.9) in the ${ }^{13} \mathrm{C}-\mathrm{NMR}$ spectrum which were absent in spectrum of niazirin added credence to the position. Other observations are as rightly indicated for niazidin. Thus, the structure of niazirinin, 4-[(4'-O-acetyl-a-Lrhamnosyloxy)benzyl] nitrile was established as the 4'-acetyl derivative of niazirin, a nitrile glycoside.

The structural elucidation of compound $\mathbf{7}$ was based on the ${ }^{1} \mathrm{H}-\mathrm{NMR}$ and ESI-MS data generated in conjunction with the infrared data which were compared to data reported in the literature (26, 27). The molecular ion $[\mathrm{M}+\mathrm{H}]^{+}$peak at $\mathrm{m} / \mathrm{z} 425.11$ in the mass spectrum confirms the molecular weight of the compound which corresponding to $\mathrm{C}_{14} \mathrm{H}_{18} \mathrm{NO}_{10} \mathrm{~S}_{2}^{-}$. The agylcone moiety with protons resonating at 7.18 and 7.02 with a coupling constant of $8.4 \mathrm{~Hz}$ supports a para-substituted benzene structure. The infrared spectrum affirms the presence of the major functional groups at 3412, 1610 and 1384 corresponding to $\mathrm{N}-\mathrm{H}, \mathrm{C}=\mathrm{C}$ and $\mathrm{S}=\mathrm{O}$ stretching vibrations. The $\mathrm{C}=\mathrm{N}$ of the glucosinolate was observed at $1645 \mathrm{~cm}^{-1}$. With comparison with literature data, the compounds were established as 4-(-a-L-rhamnopyranosyloxy) benzyl glucosinolate also called glucotropaeolin. Glucotropaeolin has been isolated from the Moringa oleifera and Bretschneidera sinensis (28). It is also known to occur in many edible plants that include broccoli and cabbage (22, 29). The glucosinolates are water-soluble glycosides which constitute an interesting class of natural products with its aglycone containing nitrogen and sulphur. A class of the glucosinolates known as benzylglucosinate (glucotropaeolin) is presumed to be the precursor of the benzyl isothiocyanate (30). The toxicity of glucosinolates is not fully established but evidence of the potential anticancer activities exist in literature $(31,32)$.

Compound $\mathbf{8}$ obtained as a viscous oil was characterised using the infrared, mass spectrometry, ${ }^{1} \mathrm{H}-\mathrm{NMR}$ and ${ }^{13} \mathrm{C}-\mathrm{NMR}$ data and comparison with the literature (33-35). Precisely, the infrared spectrum showed characteristic absorption bands 2926, 1747 and $1610 \mathrm{~cm}^{-1}$ corresponding to the $\mathrm{C}-\mathrm{H}, \mathrm{C}=\mathrm{O}$ (carbonyl of esters) and $\mathrm{C}=\mathrm{C}$ (olefin) along with stretchings of the tryglycerol. The absence of the $\mathrm{O}-\mathrm{H}$ stretching at 3200 - $3600 \mathrm{~cm}^{-1}$ confirms that the absence of fatty acid but instead, an ester. The presence of the carbonyl chemical shift at $173.3 \mathrm{ppm}$ (typical of esters) in the ${ }^{13} \mathrm{C}-\mathrm{NMR}$ spectrum attests to the claim. The molecular weight of the compound was depicted by the $\left[\mathrm{M}+\mathrm{NH}_{4}\right]^{+}$observed at $\mathrm{m} / \mathrm{z} 903.47$ $\left[\mathrm{M}+\mathrm{NH}_{4}\right]^{+}$which corresponds to the molecular formula $\mathrm{C}_{57} \mathrm{H}_{104} \mathrm{O}_{6}$. In the ${ }^{1} \mathrm{H}-\mathrm{NMR}$, the equivalent terminal methyl protons were observed between 0.88-0.90 ppm as triplets while methylene protons were clearly depicted at $1.25-1.30,1.60 \mathrm{ppm}$. The chemical shift at 5.39 and 5.23 ppm confirms the presence of the vicinal methine protons (olefin) and the methine proton of the glycerol backbone. Furthermore, the ${ }^{13} \mathrm{C}-\mathrm{NMR}$ spectrum also indicated the chemical shifts typical of $\mathrm{C}=\mathrm{C}(129 \mathrm{ppm})$ and triglycerol backbone at 69.1 and $62.2 \mathrm{ppm}$ (33). The compound was thus identified as triolein $\mathbf{8}$, a triglyceride. Triolein have been reportedly identified in the plants previously (25).

The structure of compound $\mathbf{9}$ was established by using the infrared, mass spectrometry, ${ }^{1} \mathrm{H}-\mathrm{NMR}$ and ${ }^{13} \mathrm{C}-\mathrm{NMR}$ data and comparison with literature $(33,34)$. However, the infrared and ${ }^{1} \mathrm{H}-\mathrm{NMR}$ data were quite identical with that of compound $\mathbf{8}$ with minor differences. The major difference was observed in the ESI-MS spectrum where an additional fragment peak at $\mathrm{m} / \mathrm{z} 824$ observed for $\left[\mathrm{M}^{+}-\mathrm{C}_{9} \mathrm{H}_{18}\right]^{+}$corresponding to $\mathrm{C}_{48} \mathrm{H}_{86} \mathrm{O}_{6}$ fragment was observed. This fragment which was not observed in the triolein is supposedly as a result of cleavage of the $\mathrm{C}=\mathrm{C}$ bond at position 7 from the lipidic tail. It was reported that the slight shift in the chemical shift of the carbonyl and olefinic carbon signals in the C-13 NMR are sufficient to distinguish between the triolein and the trivaccenin (34). The hydrolysis of the triolein yields omega-9 fatty acids while the trivaccenin hydrolysis yields omega-7 fatty acids both liberating the glycerol. The omega-7 and omega-9 fatty acids obtained in the Moringa oleifera seed are both monounsaturated fatty acids that are found in various other animal fats and plant oils and reported to possess various important pharmacological potentials (36).

The GC-MS analysis of the major fraction of the ethanol extract depicted ten peaks corresponding to ten known natural compounds (Table 1). Our previous report indicated that oleic acid is the most abundant fatty acid in the seed oil of Moringa oleifera [10]. The fatty acid profile reported is in agreement with other reports $(2,4)$. The fatty 
acids which include the stearic acid, palmitic acid and vaccenic acid (5) have been reported as part of the fatty acid profile of the seed. The presence of monopalmitin is suspected to be from the partial enzymatic hydrolysis of palmitin triglyceride while the methyl trans-vaccenate could also be hydrolysis product of trivaccenin reported as compound 9 in this report. The a -Irhamnopyranose and p-hydroxybenzyl cyanide are likewise rationalised to be hydrolysis products of niazirinin (compound 6), a 4-[(4'-O-acetyl-a-Lrhamnosyloxy)benzyl] nitrile also reported in this work. The GC-MS result of the fraction was further corroborated by the infrared data obtained. The FT-IR spectrum of the fraction revealed the presence of prominent $\mathrm{O}-\mathrm{H}$ stretching vibration at $3392 \mathrm{~cm}^{-1}$ and $\mathrm{C}-\mathrm{H}$ of aliphatic hydrocarbon at 2926 and $2854 \mathrm{~cm}^{-1}$. Interestingly, the cyanide stretching vibration apparently from the hydroxybenzyl cyanide was observed at $2173 \mathrm{~cm}^{-1}$. The carbonyl of esters and $\mathrm{C}=\mathrm{C}$ stretching vibration of olefins were significantly represented at 1716 and $1612 \mathrm{~cm}^{-1}$ respectively. The complexity of the infrared spectrum indicated that the fraction is a mixture of compounds with numerous functional groups.

The compounds identified in this work belonged to the thiocarbamate, isothiocyanate, nitrile, glucosinolate and lipid class of naturally occurring compounds and are presumably responsible for the primary biological activities observable in the seed. Additionally, the in silico evaluations revealed that compounds which include niazinin triacetate and trivaccenin are probable immunotoxic, carcinogenic, and mutagenic compounds on the computational toxicity models. Most of the compounds were predicted as cytochrome 3A4 substrate and/or inhibitors with good probabilities. The seed of $M$. oleifera has been implicated for some measure of toxicities in various models (37, 38). This result partly validates the previous report of the potential toxicity of the $M$. oleifera seeds (39).

\section{CONCLUSION}

In this work, a comprehensive phytochemical investigation of the seed of $M$. oleifera of Nigerian origin has been carried out and nine compounds comprising of niazimicin $\mathbf{1}$, aiazidin $\mathbf{2}$, glucomoringin isothiocyanate $\mathbf{3}$, niazinin acetate $\mathbf{4}$, niazinin triacetate $\mathbf{5}$, niazirinin $\mathbf{6}$, glucotropaeolin $\mathbf{7}$, triolein $\mathbf{8}$ and trivaccenin $\mathbf{9}$ have been characterised. Ten other compounds were identified from the GC-MS of a fraction obtained from the column chromatography. Oleic acid, 13docosenoic acid, and stearic acid were the major compounds in the fraction. All the nineteen compounds identified thus far belong to the thiocarbamate, isothiocyanate, nitrile, glucosinolate, and lipid class of naturally occurring compounds. The in silico studies implicated niazinin triacetate and trivaccenin as possible immunotoxic, carcinogenic, and mutagenic compounds indicating the need to take caution in the consumption of the seed. The report thus obtained serves as a basis for the evaluation of the pharmacological actions and toxicities of the seed.

\section{ACKNOWLEDGEMENTS}

Authors are grateful to the Raw Materials Research and Development Council, Abuja, Nigeria for the financial assistance with grant number RMRDC/AF/ 20/S.102/1.

Conflict of Interest: Authors have no conflict of interest.

\section{REFERENCES}

1. Sastari BN. The Wealth of India, Council of Scientific and Industrial Research, New Delhi, 1962 ; p. 425.

2. Liu Y, Wang X, Wei X, Gao Z, Han J. Values, properties and utility of different parts of Moringa oleifera: an overview. Chinese Herbal Medicines. 2018; 10 (4): 371-378.

3. Ravani A, Prasad RV, Gajera RR, Joshi DC. Potentiality of Moringa oleifera for food and nutritional security - a review, Agric. Rev. 2017; 38: $228-232$.

4. Tiloke C, Anand K, Gengan, RM, \& Chuturgoon AA. Moringa oleifera and their phytonanoparticles: Potential antiproliferative agents against cancer. Biomedicine \& Pharmacotherapy. 2018; 108, 457466.

5. Falowo $A B$, Mukumbo $F E$, Idamokoro $E M$, Lorenzo JM, Afolayan AJ, Muchenje V. Multifunctional application of Moringa oleifera Lam. in nutrition and animal food products: A review. Food Research International. 2018; 106, 317-334.

6. Atolani O, Olabiyi ET, Issa AA, Azeez HT, Onoja EG, Ibrahim SO, Zubair MF, Oguntoye OS, Olatunji $\mathrm{GA}$. Green synthesis and characterization of natural antiseptic soaps from the oils of underutilized tropical seed. Sustainable Chemistry and Pharmacy. 2016; 4: 32-39.

7. Faizi S, Siddiqui BS., Saleem R, Siddiqui S, Aftab K, Gilani A. Isolation and Structure Elucidation of New Nitrile and Mustard Oil Glycosides from Moringa oleifera and Their Effect on Blood Pressure. J. Nat. Prod., 1994, 57 (9), 1256-126.

8. Faizi S, Siddiqui BS, Saleem R, Noor F, Husnain S. Isolation and Structure Elucidation of a Novel Glycoside Niazidin from the Pods of Moringa oleifera J. Nat. Prod. 1997, 60, 1317-1321.

9. Dayrit FM, Alcantar AD, Villasenor IM. Studies on Moringa oleifera seeds, the antibiotic compound and its deactivation in aqueous solution. Philippine Journal of Science. 1990; 119: 23. 
10. Atolani O, Olorundare OE, Anoka AN, Osin AO, Biliaminu SA. Antioxidant, Proteinase Inhibitory and Membrane Stabilization Potentials of Moringa oleifera Seed Oil. FABAD Journal of Pharmaceutical Sciences. 2018; 43 (2), 1-13.

11. Fahey JS. Moringa oleifera: A review of the medical evidence for its nutritional, therapeutic, and prophylactic properties. Trees for Life Journal. 2005; $1: 5$.

12. Kambizi L, MT Bakare-Odunola AT, Oladiji AT, Kola-Mustapha TO, Amusa O, Atolani NS, Njinga, A.L. Quadri. Proteinease inhibition, membrane stabilization, antioxidant and phytochemical evaluations of leaves, seeds and calyces of four selected edible medicinal plants. Cogent Chemistry. 2017: 3: 1314064.

13. Şanli A, Tahsin KT. Geographical impact on essential oil composition of endemic Kundmannia anatolica Hub.-Mor. (Apiaceae). African Journal of Traditional, Complementary and Alternative Medicines. 14; (1): 131-137.

14. Njan AA, Atolani O, Olorundare OE, Afolabi SO, Ejimkonye BC, Crucifix PG, Salami B, Olajide JO, Oyewopo AO. Oyeleke SA. Chronic toxicological evaluation and reversibility studies of Moringa oleifera ethanolic seed extract in Wistar rats. Tropical Journal of Health Sciences. 2018; 25 (1), 59-71.

15. Olorundare OE, Bello MK, Billiaminu SA, Babatunde SA, Ibrahim OK, Njan AA. Acute and Subacute Toxicity of Defatted Ethanolic Extract of Moringa oleifera Seed in Albino Rats West. Afr. J. Pharmacol. Drug Res. 2015; 30:46-51.

16. Njan AA, Atolani O, Olorundare OE, Afolabi SO, Ejimkonye BC, Crucifix PG, Salami B, Olajide JO, Oyewopo AO. Assessment of developmental toxicity of Moringa oleifera (seed) in pregnant wistar rats. Centrepoint Journal (Science Edition), 2017; 23 (1): 1-18.

17. Banerjee P, Eckert AO, Schrey AK, Preissner R. ProTox-II: a webserver for the prediction of toxicity of chemicals. Nucleic Acids Research, $2018 ; 46,257-263$.

18. Faizi $S$, Siddiqui BS, Saleem $R$, Siddiqui $S$, Aftab K, Gilani A. Isolation and structure elucidation of novel hypotensive agents, Niazinin A, Niazinin B, Niazimicin and Niaziminin A + B from Moringa oleifera: The first naturally occurring thiocarbamate. Journal of the Chemical Society Perkin Transactions. 1992; 1: 3237- 3241.

19. Faizi S, Siddiqui BS, Saleem R, Siddiqui $S$, Aftab K. Gilani AUH. Fully acetylated carbamate and hypotensive thiocarbamate glycosides from Moringa oleifera. Phytochemistry. 1995; 38(4), 957-963.
20. Ragasa CY, Levida RM, Don MJ, Shen CC. Cytotoxic isothiocyanates from Moringa oleifera Lam. Philippine Science Letters. 2012; 5(1):46-52.

21. de Graaf R.M., Krosse S, Swolfs AEM., Brinke E, Prill N, Leimu R, Galen PM, Wang Y., Aarts MGM. and Dam NM. Isolation and identification of 4-arhamnosyloxy benzyl glucosinolate in Noccaea caerulescens showing intraspecific variation. Phytochemistry. 2014; 110: 166-171.

22. Fahey JW, Zalcmann AT, Talalay P. The chemical diversity and distribution of glucosinolates and isothiocyanates among plants. Phytochemistry. 2001: 56(1), 5-51.

23. Kjær A, Malver O, El-Menshaw B, Reisch J,. Isothiocyanates in myrosinase-treated seed extracts of Moringa peregina. Phytochemistry. $1979 ; 18,1485-1487$.

24. Waterman $C$, Cheng DM, Rojas-Silva $P$, Poulev A, Dreifus J, Lila MA, Raskin I. Stable, water extractable isothiocyanates from Moringa oleifera leaves attenuate inflammation in vitro. Phytochem 2014; 103:114-122.

25. Ragasa $\mathrm{CY}, \mathrm{Ng}$ VAS, Shen CC. Chemical Constituents of Moringa oleifera Lam. Seeds. International Journal of Pharmacognosy and Phytochemical Research. 2016; 8(3); 495-498.

26. Lamar S. and Rollin P. Synthesis of an Artificial Phosphate Bio-isostere of Glucotropaeolin. Tetrahedron Letters, 1994; 35, 14: 2173-2174.

27. Aucagne V, Gueyrard D, Tatibouet A, Cottaz S, Driguez $H$, Lafosse $M$ and Rollin $P$. The first synthesis of C-glucotropaeolin. Tetrahedron Letters 1999; 40: 7319-7321.

28. Montaut S, Zhang W, Nuzillard, J., De Nicola G.R., Rollin P. Glucosinolate Diversity in Bretschneidera sinensis of Chinese Origin. Journal of Natural Products. 2015; 78 (8): 2001-2006.

29. Ishida $M$, Hara M. Fukino, N; Kakizaki, T; Morimitsu, Y. "Glucosinolate metabolism, functionality and breeding for the improvement of Brassicaceae vegetables". Breeding Science. 2014; 64 (1): 48-59.

30. Agerbirk N, Olsen CE. "Glucosinolate structures in evolution". Phytochemistry. 77: 16-45.

31. Samuni BM, Arad Z, Dearing MD, Gerchman Y, Karasov WH, Izhaki I. "Friend or foe? Disparate plant-animal interactions of two congeneric rodents". Evolutionary Ecology. 27 (6): 10691080.

32. Fuentes F, Paredes-Gonzalez $X$, Kong AN. Dietary Glucosinolates Sulforaphane, Phenethyl Isothiocyanate, Indole-3-Carbinol/3,3'Diindolylmethane: Anti-Oxidative

Stress/Inflammation, Nrf2, 
Epigenetics/Epigenomics and in Vivo Cancer Chemopreventive Efficacy". Current Pharmacology Reports. 1 (3): 179-196.

33. Singh B, Agrawal PK, Thakur RS. (1989). Long chain esters of Aesculus indica. Journal of Natural Products. 2015; 52 (1): 180-183.

34. Vlahov G, Chepkwony PK, Ndalut PK. 13C NMR Characterization of Triacylglycerols of Moringa oleifera Seed Oil: An Oleic-Vaccenic Acid Oil. J. Agric. Food Chem. 2002; 50 (5): 970-975.

35. Díaz M, Gavín JA, Andrade JB. Structural characterization by Nuclear Magnetic Resonance of ozonized triolein. Grasas Aceites. 2008; 59: 274281.

36. Vessby $B$, Uusitupa $M$, Hermansen $K$, Riccardi G, Rivellese AA, Tapsell LC, Nälsén C, Berglund L, Louheranta A, Rasmussen BM, Calvert GD, Maffetone A, Pedersen E, Gustafsson IB, Storlien LH. "Substituting dietary saturated for monounsaturated fat impairs insulin sensitivity in healthy men and women: The KANWU Study". Diabetologia. 2001; 44 (3): 312-319.

37. Kavitha C, Ramesh M, Kumaran S. S., and Lakshmi SA. Toxicity of Moringa oleifera seed extract on some hematological and biochemical profiles in a freshwater fish, Cyprinus carpio. Experimental and Toxicologic Pathology. 2012; 64(7-8), 681-687.
38. Ayotunde EO, Fagbenro OA, Adebayo OT, Amoo AI. Toxicity of aqueous extracts of drumstick Moringa oleifera seeds to Nile tilapia Oreochromis niloticus, fingerlings and adults. In: Proceedings of 6th international symposium on tilapia; 2004.

39. Kim Y, Jaja-Chimedza A, Merrill D, Mendes O, Raskin I. A 14-day repeated-dose oral toxicological evaluation of an isothiocyanate-enriched hydroalcoholic extract from Moringa oleifera Lam. seeds in rats. Toxicology Reports. 2018: 5, 418-426. 